\title{
The Effect of Imperfect Interface on the Dynamic Behavior of Ceramic Particles Reinforced Metal Matrix Composites
}

\author{
Yunlong Ding ${ }^{1}$, Lisheng $\mathrm{Liu}^{1,2}$, Qiwen $\mathrm{Liu}^{1}$, Jingtao Chen ${ }^{1}$, Dongfeng $\mathrm{Cao}^{2}$, Zhen Wang ${ }^{1}$
}

\author{
${ }^{1}$ Department of Engineering Structure and Mechanics, School of Science, Wuhan University of \\ Technology, 122 Luoshi Road, Wuhan, 430070,P.R. China whut_dingyunlong@ 163.com \\ ${ }^{2}$ State Key Laboratory of Advanced Technology for Materials Synthesis and Processing, Wuhan \\ University of Technology, 122 Luoshi Road, Wuhan 430070,P.R. China liulish@whut.edu.cn
} \begin{abstract}
A new voronoi representative volume element (RVE) model with Cohesive Zone Models is advanced to investigate the effect of the interfacial properties on the dynamic behavior of ceramic-metal composites in this paper. This method is the combination of RVE with random multi-particles based on voronoi diagram and cohesive zone models which is used to describe interfacial mechanical behavior. Two kinds of interface are considered in this work, one is perfact interface; the other is weak interface which is imperfect(including the strong and weak interface). The mechanical behaviors imperfect interface are described by classical Cohesive Zone Models. By this method, the effect of interface on the dynamic behavior of particle reinforced metal matrix composites had been investigated. The numerical results show that the interface properties have huge impact on dynamic mechanical behavior of composites, strong interfaces will delay the failure of marix; however, the composites will fail rapidly after the interfaces failed while the interfaces are weak.
\end{abstract}

Keywords: particle reinforced metal matrix composites, the representative volume element, voronoi diagram, cohesive zone element, dynamic behavior

\section{Introduction}

Because the particle reinforced metal matrix composites (PRMMCs) is made of ceramic phase and metal phase, it becomes a kind of materials with some unique characteristics, such as light weight, high specific strength, high specific modulus, low thermal-expansion coefficient and good wear resistance. Theses characteristics make PRMMCs become the best candidate for application in some extremely condition, such as impact loading, extremely high temperature and etc. Nevertherless, some fatal defects in PRMMCs may lead to the disastrous failure of the PRMMCs structure if PRMMCs is not properly taken care. In fact, it becomes more serious when the PRMMCs structure suffers the dynamic loading.

Experiment, numerical simulation and theory analysis have been carried out to make clear how the defects, especially interface defects, change the PRMMCs' dynamic behaviors. At the very begining, the researchers investigated it mainly by expeimental methods. Until the computers have been applied widely, many numerical methods had been advanced to try to make clear it. These methods are proved to be very effective and economic, especially, micro-mechanics methods are a class of typical representatives of these methods because the researchers can get the dynamic behavior of PRMMCs by the representive volume element (RVE) of PRMMCs. Most of the traditional RVE model adopt axisymmtric unit cell model to predict the dynamic properties of PRMMCs. However, these models cannot reflect the effect of both particle distribution and interface properties on the damage and dynamic properties of PRMMCs. The work of Zhang[1] proved if the proper damage mechanisms and the dynamic mechanical properties can be obtained by a multi-particles model with different particles distribution and different particle/matrix interfaces, the dynamic properties of composites can be predicted accurately. This means the interface must be considered if we want to get more preciously predictive results about the dynamic behavior of PRMMCs.

The Cohesive Zone Models (CZMs) is a successful method to describe the mechanical behavior of interfaces. The concept of CZMs was first proposed by Barenblatt in 1959, and now CZMs are widely used to simulate the propagation of cracks and interfaces' failure for different materials.

In this paper, a new voronoi representative volume element(VRVE) model with Cohesive Zone Models is advanced to investigate the effect of the interfacial properties on the dynamic behavior of ceramic-metal composites. a VRVE model is created by the following steps. The size of RVE must chose firstly, then the RVE is divided into Voronoi diagram according to the nearest-neighbor rule [2], and some polygons are assigned to ceramic's material properties according to the specific volume fraction of ceramic phase and uniform random distribution of particle size, finally, a VRVE model can be obtained when the finite element meshes on VRVE are generated. According to the above method, the micro-mechanical models are built (with different volume fraction and different interfical properties) to study the dynamic behavior of PRMMCs. 


\section{Multi-particles vornoi representative volume element (VRVE) with cohesive zone interface}

\subsection{The interface model-cohesive zone interface}

Two constitutive model of CZMs are now widely used, exponential model[3] and bilinear model[4]. In this paper, the bilinear constitutive model is used for simulating the interface between metal and matrix. The interfacial constitutive relations of traction force-displacement for normal and tangential is shown in Fig 1. (a) and (b)[5].

\subsection{Multi-particles vornoi representive volume element(VRVE)}

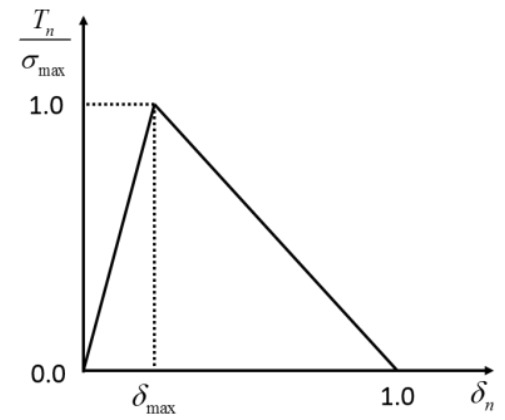

(a)

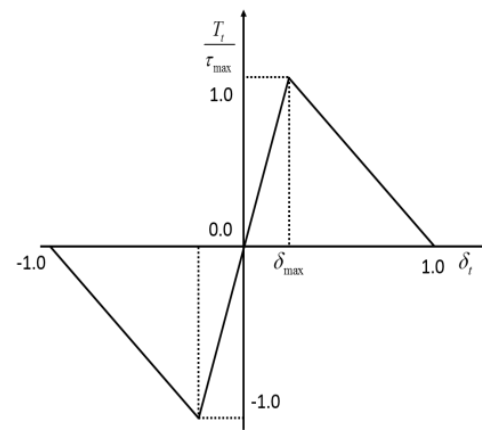

(b)

Fig. 1. The relationship between traction force and displacement, (a) Normal traction $T_{n}$, (b) shear traction $T_{t}$

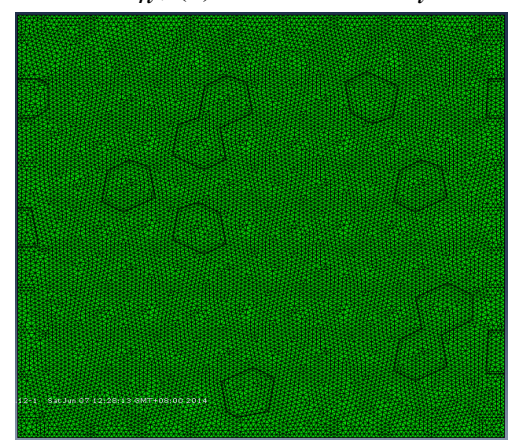

(a)

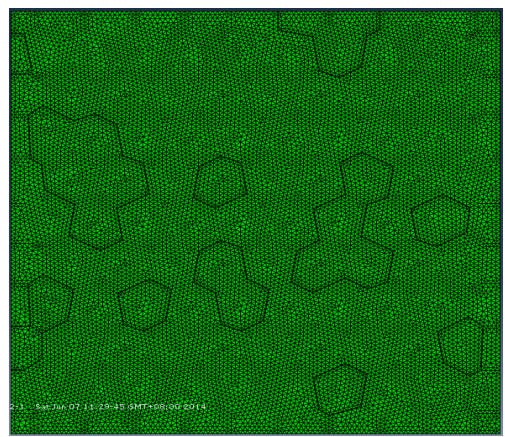

(b)

Fig. 2. FEM with CZMs, (a) the particles volume fraction is $10 \%$, (b) the particles volume fraction is $20 \%$

about $0.01 \mathrm{~mm}$, the total model contains 25768 elements without CZMs.

The cohesive elements between particles and matrix are generated by a program, the program gets nodes and elements information from files firstly, then finds the common edges, adds new nodes and moves the node by a parameter $\mathrm{u}$. By doing these operations, the CZMs elements with a thickness of $0.1 \times \mathrm{u} \mathrm{mm}$ are generated. The models with CZMs are shown in Fig. 2.

The Johnson-Cook material model is used for the Al matrix, Elastic material model for the SiC particles and bilinear CZMs for the interfaces. The parameters used in this paper are listed in the Table 1 and Table 2.

Table. 1 Material properties[6]

\begin{tabular}{|c|c|c|c|c|}
\hline \multirow[b]{2}{*}{ Material } & \multirow[b]{2}{*}{ Poisson's ratio } & \multirow[b]{2}{*}{ Young's Modulus } & \multicolumn{2}{|c|}{ Cohesive properties } \\
\hline & & & $\begin{array}{l}\text { Cohesive Energy } \\
\phi_{\max }\left(\mathrm{J} / \mathrm{m}^{2}\right)\end{array}$ & $\begin{array}{l}\text { Cohesive Strength } \\
\sigma_{\max }(M P a)\end{array}$ \\
\hline $\mathrm{SiC}$ & 0.17 & 427 & & \\
\hline Al matrix & 0.33 & 76 & & \\
\hline Interface & & 76 & 91.9 & 430 \\
\hline
\end{tabular}

Table. 2 Johnson-Cook strength and fracture model constants for aluminum used in the computations $[7,8]$

\begin{tabular}{|c|c|c|c|c|c|c|c|c|c|c|c|c|}
\hline \multirow{2}{*}{ Material } & \multirow{2}{*}{$\begin{array}{l}\text { Density } \\
\rho\left(\mathrm{kg} / \mathrm{m}^{3}\right)\end{array}$} & \multirow{2}{*}{$\begin{array}{l}\text { Shear Modeulus } \\
G(\mathrm{GPa})\end{array}$} & \multicolumn{5}{|c|}{ Strength constants } & \multicolumn{5}{|c|}{ Fracture constants } \\
\hline & & & $\mathrm{A}(\mathrm{MPa})$ & $\mathrm{B}(\mathrm{MPa})$ & $\mathrm{N}$ & $\mathrm{C}$ & $\mathrm{M}$ & $D_{1}$ & $D_{2}$ & $D_{3}$ & $D_{4}$ & $D_{5}$ \\
\hline $\begin{array}{l}6061-\mathrm{T} 6 \\
\text { Aluminum }\end{array}$ & 2704 & 28 & 324 & 114 & 0.42 & 0.002 & 1.34 & -0.77 & 1.45 & -0.47 & 0.00 & 1.60 \\
\hline
\end{tabular}

Presenting and corresponding author: Master Candidate, Yunlong Ding, P.R. China 


\subsection{Boundry Conditions}

The boundry conditions of FEM VRVE need be met the basic conditions of a RVE. The periodic boundary conditions must be applied firstly for RVE model. To get this point, the specimen's left and right sides must remain straight by coupling the nodes displacement in horizontal direction; the vertical displacement of the specimen's bottom side must be constrained; the displacement of the nodes on the tope side are change with time for simulating the dynamic tensile load ( Eq. (1)) [1].

$$
u(0, y)=0 \quad \text { on } x=0 ; \quad u(x, 0)=0 \quad \text { on } y=0 ; \quad \dot{u}(x, y)=\dot{u}(\mathrm{~L}, \mathrm{y}) \quad \text { on } \quad x=L ; u(x, H)=\varepsilon_{0} \times t_{i} / t \quad \text { on } \quad y=H
$$

\section{Results and Discussions}

In the simulation, time step is set as $t=0.1 \mathrm{~ms}$ for the strain rate $\dot{\varepsilon}=1500 \mathrm{~s}^{-1}$ and $t=1 \mathrm{~ms}$ for the strain rate $\dot{\varepsilon}=150 \mathrm{~s}^{-1}$. The following formulations are used to calculate the equivalent stress and strain[1]:

$$
\bar{\sigma}_{y}^{i}=\left(F_{y=0}^{i}+F_{y=H}^{i}\right) /\left(L^{i} / 2\right) ; \quad{ }^{-i} y=u_{y}^{i}(x, H)
$$

where $i$ is the $i$ th load step; $\bar{\sigma}_{y}^{i}$ is the average stress of the model; $F_{y=0}^{i}$ and $F_{y=H}^{i}$ are the sum of the nodes' reaction force on the bottom side and the top side, respectively; $L^{i}$ is the sample's width at the $i$ th load step. $\bar{\varepsilon}_{y}^{i}$ is the average strain of the model on y-direction and $u_{y}^{i}(x, H)$ is the displacement of the top side.

The true stress-strain curves under the different conditions are given in the Fig 3 and Fig.4, the stress distribution and damage are shown in Fig. 5 and 6.

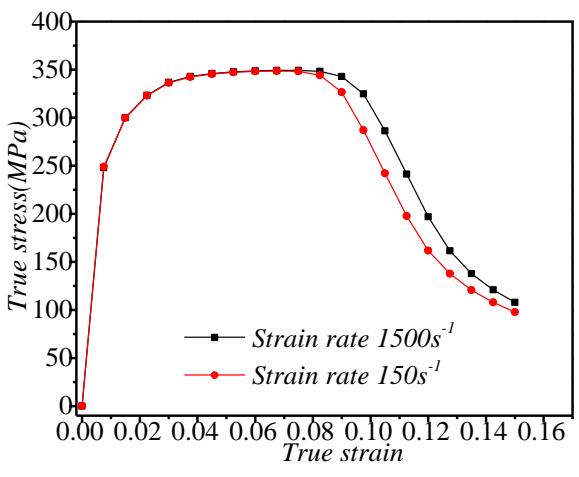

(a)

Fig. 3: The True stress-strain curve with CZMs, (a) the particles volume fraction is $10 \%$, (b) the particles volume fraction is $20 \%$

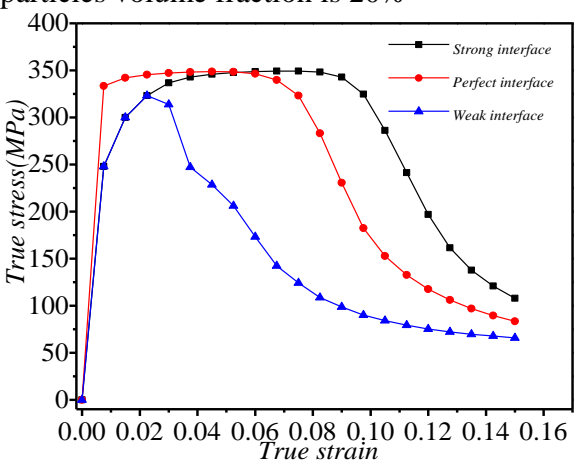

(a)

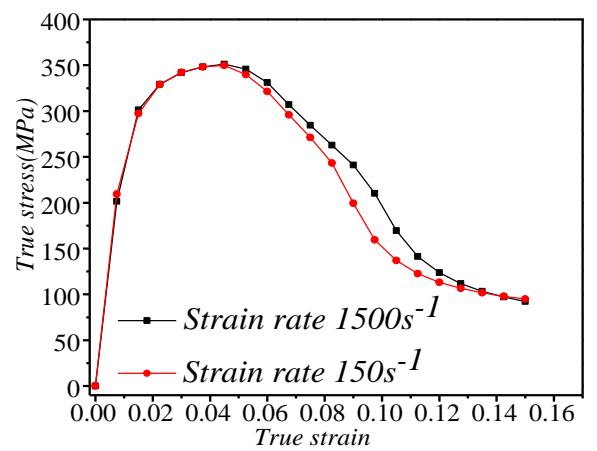

(b)

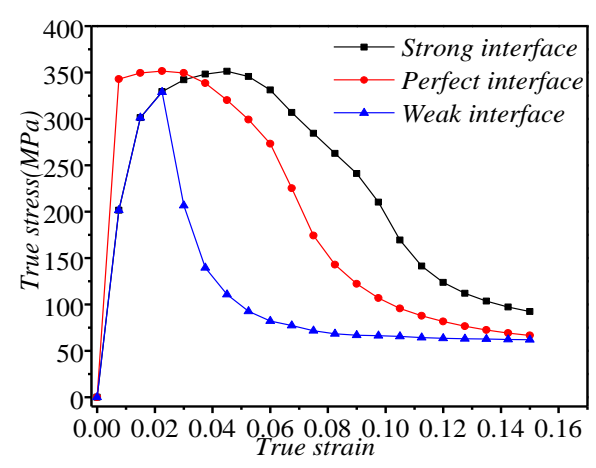

(b)
Fig. 4: The True stress-strain curve with different interfaces, $\dot{\varepsilon}=1500 \mathrm{~s}^{-1}$, (a) the particles volume fraction is $10 \%$, (b) the particles volume fraction is $20 \%$
From Fig.3 and Fig. 4, composite materials fail slower (for strain) under the interaction of the lower strain rate load than for the higher strain rates, but this phenomenon is not very obvious. So we just discuss the case for the strain-rate $\dot{\varepsilon}=1500 \mathrm{~s}^{-1}$ in the following part. Fig. 4 indicates the true stress-strain curve gets down in the end because of the yield and damage of the matrix while the composite materials fail at last. Compared with Fig.4 (a) and Fig.4 (b), the curves get down faster as the particles' volume fraction is higher, it is because for the model with the higher particles' volume fraction, the matrix has higher stress level, so the matrix will obtain the yield stress and fail sooner. It also can be found from Fig.4 (a) and (b) that the yield strength of the models without CZMs is higher than that of the models with CZMs, but the matrix of the models without CZMS failed faster than that of the models with CZMs.

From Fig.4, the elastic stage for the composite with three diffenent interfacial properties are almost the same, but the plastic stages of them are very different. That is because after the composite materials enter yield stage, for the composites with lower interfacial strength, the stress-strain curve gets down very soon after the interfaces failed; for the composites with strong interface, the true stress-strain curve gets down later.

By observing the stress and damage distribution in Fig 5 and 6, it can be found that if the strength of interfaces is higher than that of the matrix, the yield regions of the models with or without interfaces are almost same, but when the strength of interfaces is lower than that of matrix, the interfaces fail earlier than the matrix, and the damage and failed regions of different model are very different. 


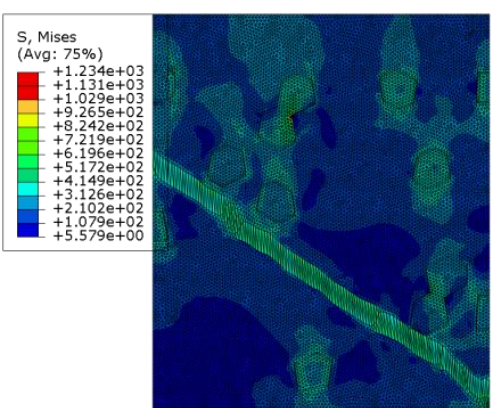

(a)

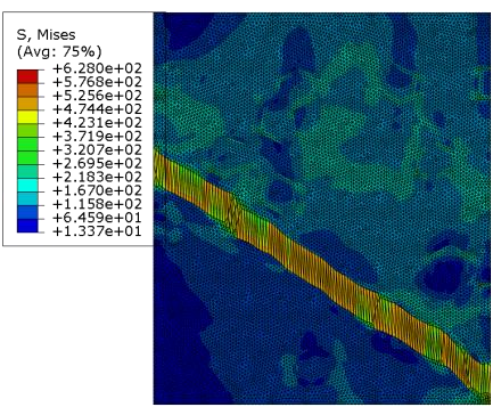

(b)

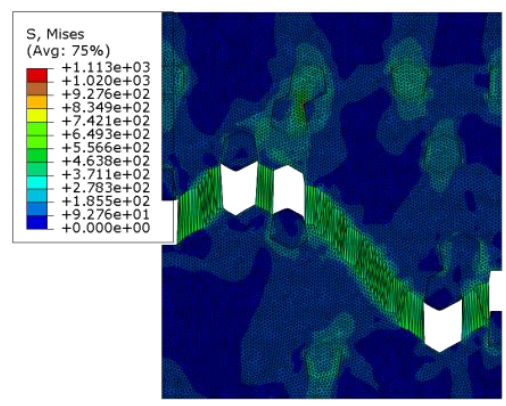

(c)

Fig. 5: the von mises stress distribution and the damage zone in VRVE( the particles volume fraction:10\%, $\dot{\varepsilon}=1500 s^{-1}$ ) $/ \mathrm{MPa}^{2}$

(a) strong interface, (b) perfect interface, (c) weak interface

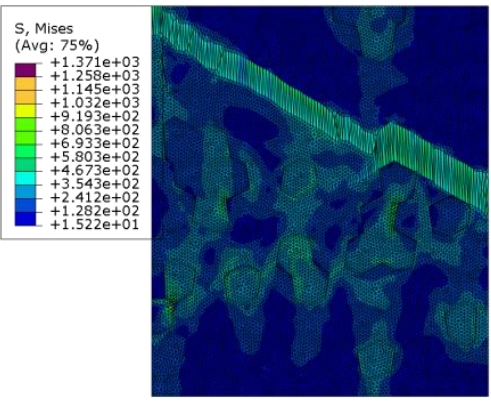

(a)

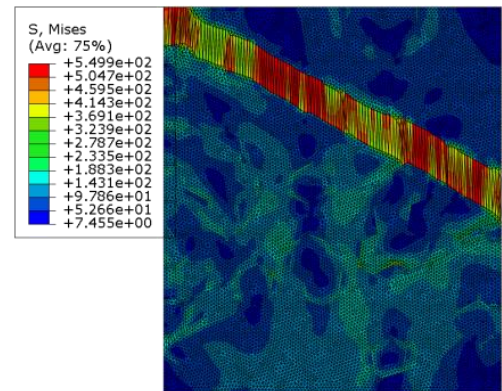

(b)

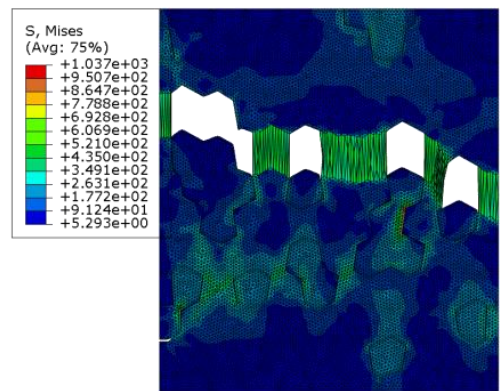

(c)

Fig. 6: the von mises stress distribution and the damage zone in VRVE( the particles volume fraction: $20 \%, \dot{\varepsilon}=1500 s^{-1}$ ) /MPa

(a) strong interface, (b) perfect interface, (c) weak interface

\section{Conclusions}

A new voronoi representative volume element(RVE) model with Cohesive Zone Models is advanced to investigate the effect of the interfacial properties on the dynamic behavior of ceramic-metal composites in this paper. This method is the combination of RVE with random multi-particles based on voronoi diagram and cohesive zone models which is used to describe interfacial mechanical behavior.

Bases on this new RPV model, the effect of of imperfect interface on the dynamic behavior of ceramic particles reinforced metal matrix composites have been investigated. Numerical results indicate:

(i) The volume fraction of the particles has great influence on the dynamic behavior of the PRMMCs: the more ceramic phase within the composites, the weaker the failure strength of the matrix is.

(ii) The strength of interface has great influence on the yield region of the PRMMCs. Strong interfaces will delay the failure of matrix, and the composites materials with the weak interfaces will fail rapidly after the failure of interfaces.

\section{Acknowledgements}

This work is financially supported by the National Program on Key Basic Research Project (973 Program, No. 2013CB632505), and the Fundamental Research Funds for the Central Universities (WUT: 2013-Ia-033 and 2013-Ia-029).

\section{References}

[1] Zhang, J., Experimental and Numerical studies on the Dynamic Mechanical Properties of Particle Reinforced Metal-Matrix Composites, 2007, Wuhan University Of Technology.

[2] Aurenhammer, F., Voronoi diagrams - a survey of a fundamental geometric data structure. ACM Computing Surveys (CSUR), 1991. 23(3): p. 345-405.

[3] Xu, X.P. and A. Needleman, Numerical simulations of fast crack growth in brittle solids. Journal of the Mechanics and Physics of Solids, 1994. 42(9): p. 1397-1434.

[4] Geubelle, P.H. and J.S. Baylor, Impact-induced delamination of composites: a 2D simulation. Composites Part B: Engineering, 1998. $29(5)$ : p. $589-602$.

[5] Chandra, N., et al., Some issues in the application of cohesive zone models for metal-ceramic interfaces. International Journal of Solids and Structures, 2002. 39(10): p. 2827-2855.

[6] Yuan, Z., et al., Analysis of the stress states and interface damage in a particle reinforced composite based on a micromodel using cohesive elements. Materials Science and Engineering: A, 2014. 589(0): p. 288-302.

[7] Holmquist, T.J., D.W. Templeton, and K.D. Bishnoi, Constitutive modeling of aluminum nitride for large strain, high-strain rate, and high-pressure applications. International Journal of Impact Engineering, 2001. 25(3): p. 211-231.

[8] Lesuer, D.R., G. Kay, and M. LeBlanc, Modeling large-strain, high-rate deformation in metals. UCRLJC-134118, Lawrence Livermore National Laboratory, 2001. 